The Egyptian Journal of Hospital Medicine (October 2018) Vol. 73(1), Page 5875-5885

\title{
Comparison between the role of contrast enhanced mammography and dynamic contrast enhanced MRI in assessment of breast cancer recurrence
}

\author{
Saher Mohamed Elfiky, Noha Abdelshafy Elsaid, Enas ahmed Azeb, Marwa Elsayed \\ Elmor, Nehal Abdelshafy Abdelaleem \\ Radiology department, Faculty of medicine, Ain Shams University \\ Corresponding Author: Tarek Hamed Gomaa Hassan, \\ Email:Tarekhamed11@ hotmail.com. Tel.01062229712
}

\begin{abstract}
Background:

Purpose: To elucidate the diagnostic value of contrast enhanced mammography in comparison with dynamic MRI in cases of breast cancer recurrence and to correlate with available clinical data, histopathology findings and/or follow up. Methods: 30 patients were enrolled in this study. The age ranged between31-68 years old. Patients were referred from the General Surgery or Oncology Departments in the period from August-2015 until April-2018. The patients underwent full history taking and clinical examination and CESM and breast magnetic resonance image (BMRI) examination. Finally US guided biopsy was performed. Results: Contrast enhanced spectral mammography (CESM) showed slightly lower sensitivity (88.89\%) and overall accuracy (86.67\%) than BMRI (96.30\% and 90\% respectively). However specificity was higher in CESM (66.67\%) than that of BMRI (33.33\%). Conclusion: In spite of the lower sensitivity of the CESM compared to MRI, the CESM appeared to be a suitable, easy, more comfortable, low cost and fast alternative to MRI in early detection of breast cancer recurrence specially for patients with contraindications to MRI.
\end{abstract}

Keywords: Recurrent breast cancer - BMRI - CESM.

\section{Introduction}

Breast cancer is not only a leading cause of death among women but also carries a high risk of recurrence, the earlier the diagnosis of recurrence, the better the outcome ${ }^{1}$.

Up-to-date, mammography appears to be the most consistentmethod for the early detection of breast cancer; yet, it has bothlimited sensitivity and specificity in the detection and diagnosis of breast lesions, especially in dense breasts. Moreover thefull extent of the disease may not be clearly depicted. In reference to this, mammography misses about $20 \%$ of invasive breastcancers.Therefore, management of these women should include testing that improves the early diagnosis of recurrence, as contrast enhanced mammography and $\mathrm{MRI}^{2}$.

BMRI is currently the gold standard for breast cancer detection and staging but is limited by low specificity, high cost, long duration of examination time (patient must lie still in the prone position for a half-hour or longer during image acquisition), and limited availability ${ }^{3}$.

CESM is a relatively new imaging modality that can be used as complementary test to standard mammogram using the same MG equipment, on the same examination day. Only renal function test is needed before contrast injection, than in less than 10 minutes with slightly higher exposure radiation dose, we can solve many mammographic problems with high quality image, easy to interpret by same radiologists and provides both anatomic and functional information of the lesions similar to MRI ${ }^{4}$.

\section{Patients and Methods}

Thirty patients were enrolled in this study, the age ranged between31-68 years old. Patients were referred from the General 
Surgery or Oncology Departments in the period from August-2015 until April2018.The patients underwent full history taking and clinical examination, CESM and BMRI examination.Finally us guided biopsy was performed.

\section{Each patient included in the study subjected} to:

- Full history taking.

- Reviewing medical sheet whenever available.

- Inclusion criteria:Patients with suspected recurrent breast lesion as either ipsilateral or contralateral after conservative breast surgery or modified radical mastectomy after 6 months of operative intervention.

- Exclusion criteria:a- CESM:possible pregnancy, renal impairment, or a history of allergy to a contrast agent. bMRI:contrast allergy or compromised renal function (eGFR $<30$ $\mathrm{ml} / \mathrm{min} / 1.73 \mathrm{~m} 2$ ), inability to lie prone, marked obesity, extremely large breastsand implantable devices that are not MRI compatible.

CESM protocols:The contrast agent used was the non-ionic solution (iohexol, Omnipaque 300; Nycomed, Roskilde, Denmark) containing $300 \mathrm{mg}$ of iodine per milliliter. $1.5 \mathrm{ml} / \mathrm{kg}$ of the agent was injected by hand over a period of approximately $1 \mathrm{~min}$. CESM was performed utilizingthe Senographe Essential Full Field Digital Mammographysystem equipped with CESM (SenoBright ${ }^{\circledR}, \quad$ GE, Milwaukee, Wis). CESM imageswere acquired within ten minutes after intravenous administration of contrast. CESM high and low-energy imageswere obtained in CC and MLO projections of each breast, used to produce subtracted images. CESM low-energy and subtracted images were reviewed by radiologists and the lesions were analyzed by the radiologist for the presence, morphology, and pattern of enhancement of recurrent lesions.

BMRI protocol: BMRI were performed after injection of a bolus of gadopentetatedimeglumine, in a dose of 0.2 $\mathrm{m}-\mathrm{mol} / \mathrm{kg}$ using an automated injector at a rate of $3-5 \mathrm{ml} / \mathrm{s}$ through a $18-20$ gauge intravenous cannula inserted in an antecubital vein. This was followed by a bolus injection of saline (total of $20 \mathrm{ml}$ at 3-5 $\mathrm{ml} / \mathrm{s}$ ). BMRI studies were performed using a 1.5-T MR system (on superconducting $1.5 \mathrm{~T}$ MR imaging unit (Philips Ingenia)) and a dedicated seven channel breast coil (Sentinelle BreastCoil by In vivo) after intravenously administration of contrast while patients were placed in prone position. The BMRI protocol included T1- and T2weighted pre-contrast fat suppressed images in the axial plane, dynamic imaging included one precontrast and 4 cycles of post-contrast imaging, compliant with ACRrecommendations. Reconstructed sagittal post-contrast images were also obtained.

\section{Statistics}

Using the standard of reference, sensitivity, specificity, and accuracy were calculated. In addition, comparison between CESM and BMRI groups was performed using the unpaired $t$ test and McNemartest. Correlations were sought using the Pearson correlation. A p $<0.005$ was considered significant.

\section{Results}

The 30 patients enrolled in our study, had age range between31-68 years old (Mean = $45.34 \pm 10.78$ ). The entire patienthad a history of previously surgical treated breast cancer, which either CBS (20 cases) or MRM (10 cases). Most of the recurrent lesions occurred between 1-6 years $($ Mean $=$ $3.1 \pm 1.5$ ) after the previous surgery.

By means of surgery, excision biopsy or true cut biopsy there were 27 patients with recurrent malignancy, which included 26 patients with invasive 
carcinoma (15 cases IDC and 11 cases ILC) and one case had DCIS. Three cases had benign lesion (2 cases with fibroadenoma and one case with surgical scar).

\section{Results of contrast enhanced spectral mammography (CESM):}

From 30 examined lesions, 25 lesions showed post contrast enhancement. Of these
25 cases, 24 cases were pathologically proved cancer recurrence and only one case proved to be fibroadenoma. From the 5 non enhanced cases, three cases were pathologically diagnosed to be positive recurrent lesions, one diagnosed as fibro adenoma and the other was scar. These results yielded sensitivity of $88.8 \%$, specificity of $66.67 \%$, and overall accuracy of $86.67 \%$ (table 1 ).

Table (1): Diagnostic performance of CESM

\begin{tabular}{|c|c|c|c|c|c|c|c|c|}
\hline TP & $\mathbf{F P}$ & $\mathbf{T N}$ & $\mathbf{F N}$ & Sens & Spec & PPV & NPV & Acc \\
\hline 24 & 1 & 2 & 3 & $88.89 \%$ & $66.67 \%$ & $96.00 \%$ & $40.00 \%$ & $86.67 \%$ \\
\hline
\end{tabular}

$\mathbf{T P}=$ true positive, $\mathbf{F P}=$ false positive, $\mathbf{T N}=$ true negative, $\mathbf{F N}=$ false negative, Sens = sensitivity, Spec $=$ specificity, $\mathbf{P P V}=$ positive predictive value, $\mathbf{N P V}=$ negative predictive value , Acc $=$ overall accuracy

\section{RESULTS OF MRI:}

BMRI showed enhancement in 28 cases, 26 cases were pathologically proved cancer recurrence.It showed positive enhancement in 2 case of fibroadenoma. Two cases showed no enhancement, one pathologically diagnosed to be positive recurrent lesion and the other one diagnosed as the scar.These results yielded sensitivity of $96.3 \%$, specificity of $33.33 \%$, and overall accuracy of $90 \%$ (Table 2).

Table (2): Diagnostic performance of BMRI

\begin{tabular}{c|c|c|c|c|c|c|c|c}
\hline $\mathbf{T P}$ & $\mathbf{F P}$ & $\mathbf{T N}$ & $\mathbf{F N}$ & Sens & Spec & PPV & NPV & Acc \\
\hline 26 & 2 & 1 & 1 & $96.30 \%$ & $33.33 \%$ & $92.86 \%$ & $50.00 \%$ & $90.00 \%$ \\
\hline
\end{tabular}

$\mathbf{T P}=$ true positive, $\mathbf{F P}=$ false positive, $\mathbf{T N}=$ true negative, $\mathbf{F N}=$ false negative, Sens $=$ sensitivity, Spec $=$ specificity, $\mathbf{P P V}=$ positive predictive value $\mathbf{N P V}=$ negative predictive value, $\mathbf{A c c}=$ overall accuracy

\section{RESULTS OF CESM COMPARED TOBMRI:}

\section{$\underline{\text { 1. Diagnostic performance }}$}

In this study we compared between results of CESM and BMRI using cross tabulation, it was found that there was statistically significant difference between the diagnostic performance of BMRI and CESM (table 3). 
Table (3): Final diagnosis of CESM compared to BMRI

\begin{tabular}{c|cc|c|c}
\hline \multirow{2}{*}{ BMRI } & \multicolumn{2}{|c|}{ CESM } & Total & $\begin{array}{c}\text { P-value } \\
\text { (McNemar } \\
\text { test })\end{array}$ \\
\cline { 2 - 3 } & Benign & Malignant & $2(7 \%)$ & \\
\cline { 2 - 3 } Benign & $2(7 \%)$ & $0(0 \%)$ & $28(93 \%)$ & \\
Malignant & $3(10 \%)$ & $25(83 \%)$ & & $\mathbf{0 . 0 2 3}$ \\
\hline Total & $5(17 \%)$ & $25(83 \%)$ & $30(100 \%)$ & \\
\hline \hline
\end{tabular}

CESM showed slightly lower sensitivity $\mathbf{( 8 8 . 8 \% )}$ and overall accuracy $(\mathbf{8 6 . 6 7 \%})$ than BMRI $(\mathbf{9 6 . 3 \%}$ and $\mathbf{9 0 \%}$ respectively). However specificity was higher in CESM (66.67\%) than that of BMRI (33.33\%).
Morphological Analysis of The different pattern of Lesions enhancement In CESM and BMRI.

The enhanced lesion either shows mass or non-mass enhancement the fig comparing the different patterns of enhancement in CESM and BMRI.

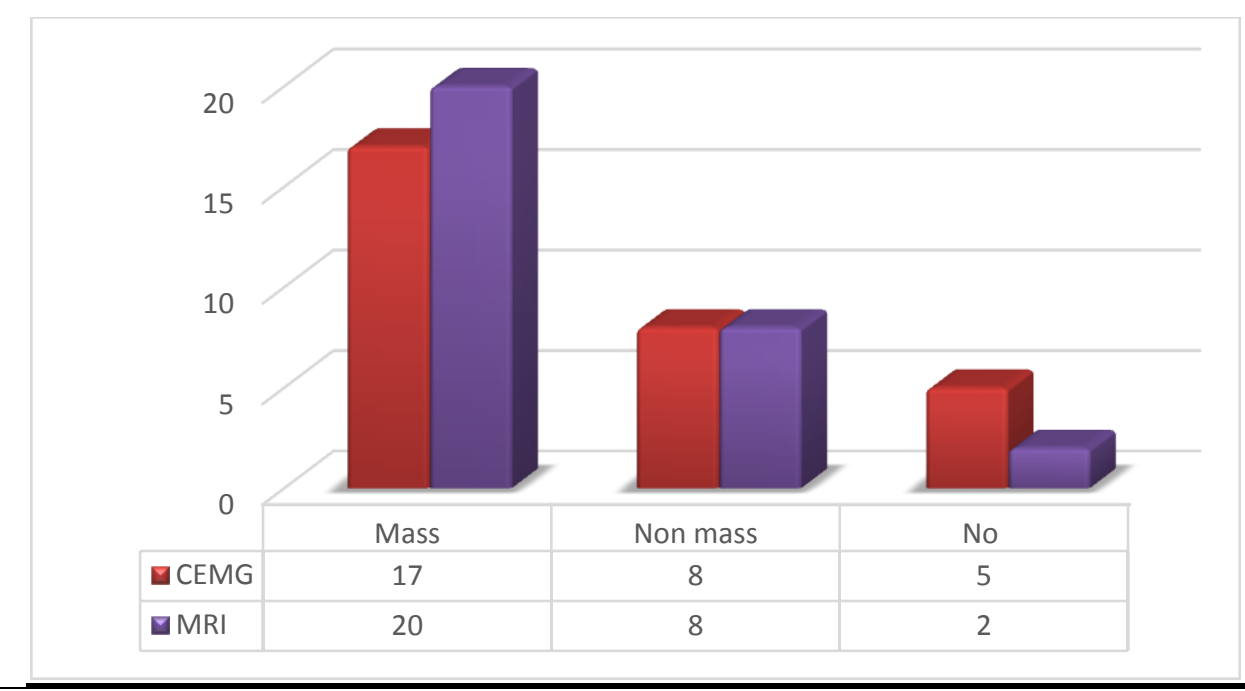

Fig (1): different enhancement pattern of recurrent lesions in CESM and BMRI

\section{a- Analysis of mass enhanced lesions parameters (table 4 )}

Analysis of mass lesion depended on mass shape, enhancement pattern, degree of enhancement and margin. This was discussed in table (4). 
Table (4): Analysis of mass enhanced lesions parameters

\begin{tabular}{ccc}
\hline & CESM & BMRI \\
\hline Mass shape & 7 & 7 \\
• Rounded/oval & 10 & 13 \\
• irregular & & \\
Enhancement pattern & 7 & 8 \\
- homogenous & 9 & 11 \\
- heterogeneous & 1 & 1 \\
- Ring pattern & & \\
Degree of enhancement & 7 & 5 \\
• faint & 10 & 15 \\
- intense & & \\
Margin & 5 & 5 \\
- Well defined & 1 & 2 \\
- Ill defined & 11 & 13 \\
- Spiculated & $\mathbf{1 7}$ & $\mathbf{2 0}$ \\
Total &
\end{tabular}

b- Analysis of non-mass enhanced lesions parameters (NME)

The NME lesion were analyzed according to its enchantment pattern, degree of enhancement and distribution of enhancement as discussed in table (5)

Table (5): Analysis of non-mass enhanced lesions parameters

\begin{tabular}{|c|c|c|}
\hline & CESM & BMRI \\
\hline \multicolumn{3}{|l|}{ Enhancement patterns } \\
\hline - homogenous & 2 & 2 \\
\hline - Heterogeneous & 6 & 6 \\
\hline \multicolumn{3}{|l|}{ Degree of enhancement } \\
\hline - faint & 2 & 1 \\
\hline - intense & 6 & 7 \\
\hline \multicolumn{3}{|l|}{ Distribution } \\
\hline - Diffuse/ patchy/nodular & 3 & 3 \\
\hline - Ductal/segmental/regional & 3 & 3 \\
\hline - focal & 1 & 1 \\
\hline - clumped & 1 & 1 \\
\hline Total & 8 & 8 \\
\hline
\end{tabular}

Mean diameter of the recurrent masses in CESM (24 lesions) was about $2.66 \mathrm{~cm} \pm$ $2.04 \mathrm{~cm}$, while mean diameter of the recurrent masses in BMRI (26 lesions) was about $2.55 \mathrm{~cm} \pm 1.95 \mathrm{~cm}$. Mean diameter of recurrent masses as found in pathology (27 lesions) was about $2.49 \mathrm{~cm} \pm 1.85 \mathrm{~cm}$.
There was no statistically significant difference regarding size estimation between CESM and pathology $(p=0.329)$, nor between MRI and pathology $(\mathrm{p}=0.607)$. Finally there was also no statistically significant difference between size estimates in CESM and MRI ( $\mathrm{p}=0.918)($ tables6,7 and 8) 
Table(6): Size estimation of recurrent lesions by CESM Compared to Pathology size.

\begin{tabular}{c|c|c|c|c}
\hline Size & $\mathbf{N}$ & Mean (cm) & SD (cm) & p-value \\
\hline CESM & 24 & 2.66 & \pm 2.04 & $\mathbf{0 . 3 2 9}$ \\
Pathology & 27 & 2.49 & \pm 1.85 & \\
\hline \hline
\end{tabular}

Table(7): Size estimation of recurrent lesions by BMRI compared to pathology size.

\begin{tabular}{c|c|c|c|c}
\hline Size & $\mathbf{N}$ & Mean $(\mathbf{c m})$ & SD $(\mathbf{c m})$ & p-value \\
\hline BMRI & 26 & 2.55 & \pm 1.95 & $\mathbf{0 . 6 0 7}$ \\
Pathology & 27 & 2.49 & \pm 1.85 & \\
\hline \hline
\end{tabular}

Table(8): Size estimation of recurrent lesions by CESM compared BMRI

\begin{tabular}{c|c|c|c|c}
\hline Size & $\mathbf{N}$ & Mean $(\mathbf{c m})$ & SD $(\mathbf{c m})$ & p-value \\
\hline CESM & 24 & 2.66 & \pm 2.04 & $\mathbf{0 . 9 1 8}$ \\
BMRI & 26 & 2.55 & \pm 1.95 & \\
\hline
\end{tabular}
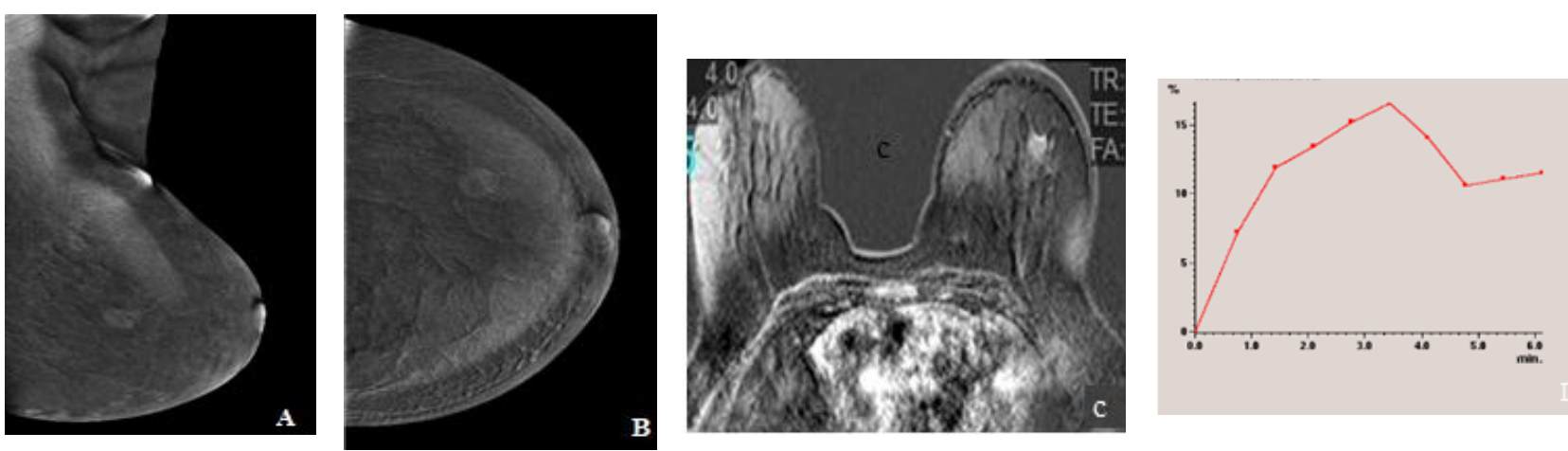

\section{CASE 1}

Fig.(2):Female patient, 57 years old, underwent left BCS one year ago followed by radiotherapy measures.CESM in (A) MLO and (B) CC views of left breast showed irregular shaped speculated mass lesion heterogeneous enhanced lesion in
LOQ.BMRI in(c) post contrast subtraction image and (D) dynamic curve of the left breast showedirregular shaped speculated mass lesion in LOQ, which elicited low T2 signal with heterogeneous enhancement post contrast series and gave type III dynamic curve.

\section{Case 2}


Fig(3):Female patient, 31 years old, underwent right radical mastectomy 2.5 years followed by left BCS 2 years ago followed by chemotherapy. She presented by left mass.CESM in (A) MLO and (B) CC views of $\mathrm{Lt}$ breast showedlarge regional NME homogenous enhanced lesion in UOQ with few enhanced foci seen in the UIQ.

B-MRI: (C) and (D) post contrast subtraction imagesand (E) dynamic curve showed the LT breast with large regional NME in UOQ which elicit rather homogenous enhancement in post contrast series that gave type III dynamic curve with few scattered foci seen at inner aspect.Associated post-operative changes in the form of thickened skin as well as skin retraction at the scar site. The RT mastectomy bed showed marked skin thickeningand multiple small enhanced foci.
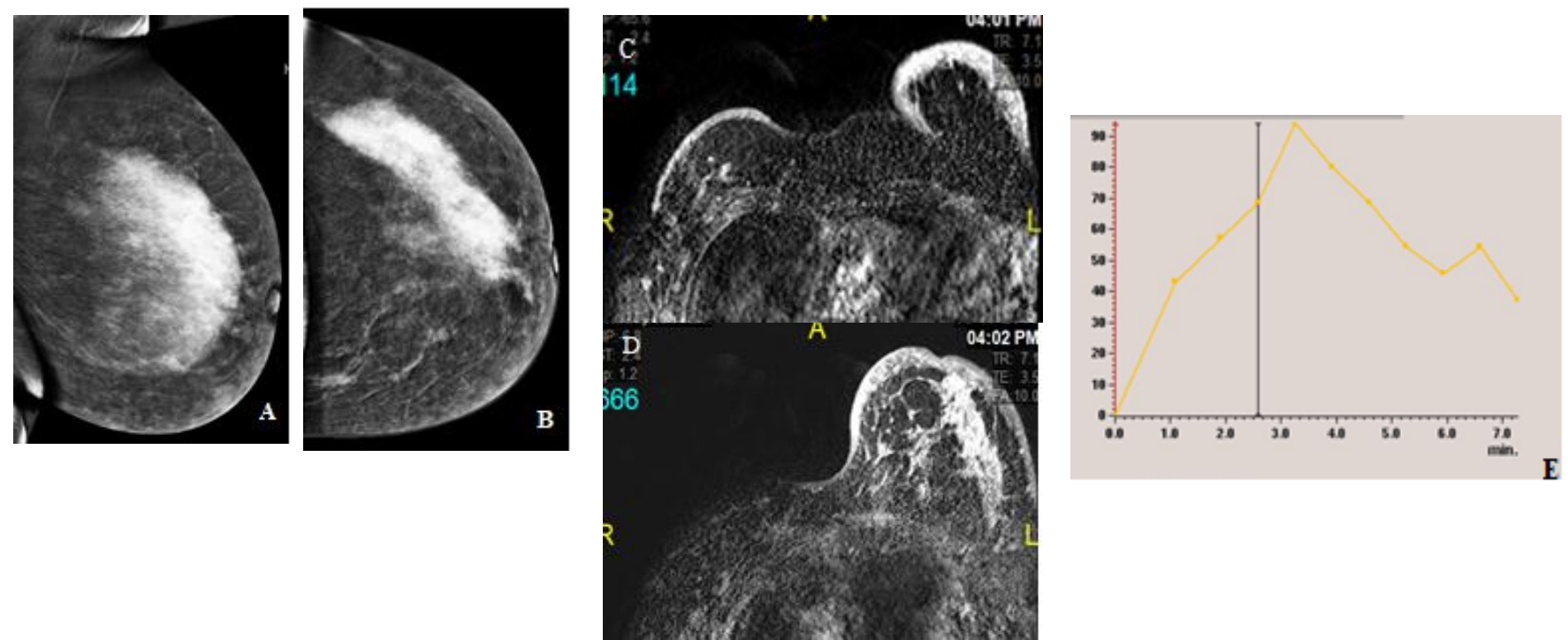

\section{Case 3:}

Fig.(4):Female patient, 54 years old, underwent right MRM followed by chemotherapy. After 3 years she presented with ipsilateral mass. CESM in (A) MLO and (B) CC views of left breast showedmulticenteric deep-seated irregular speculated homogenous enhanced mass. BMRI: (CandD)post contrast subtraction images,(E) MIP image and (F) T2 ofthe left
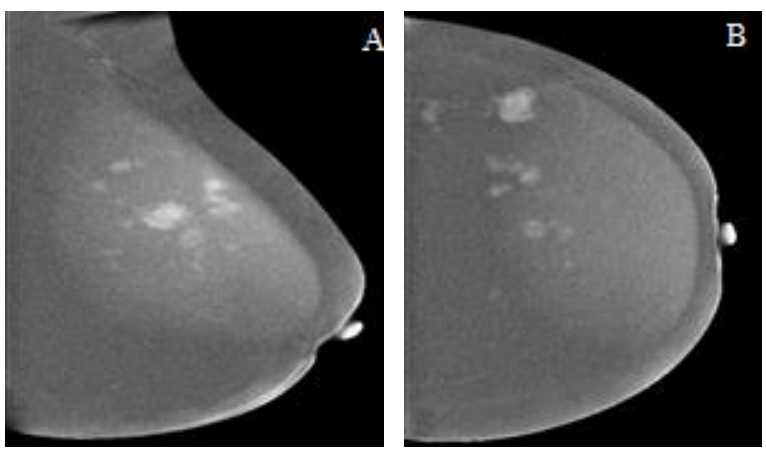

breast showedmultiple scattered variable size irregular speculated mass lesions elicitedvariable pattern of enhancement in post contrast series.Note that MIP image gave a map of these multiple. The largest lesion was in the left side. The RT mastectomy bed showed multiple smallenhanced lesions as well as skin nodules. Multiple variable size liver metastases seen at $(\mathrm{F})$.

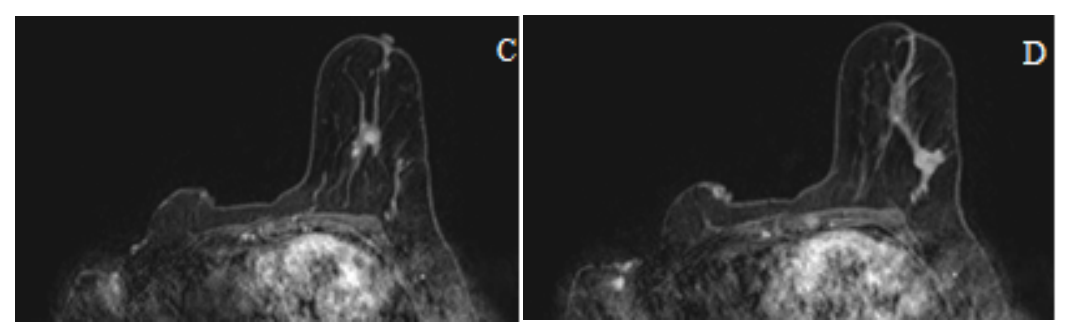




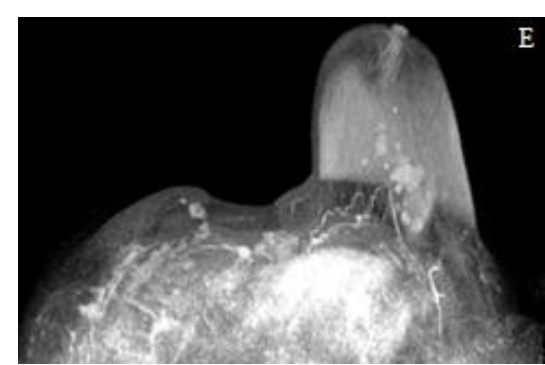

\section{Case 4}

Fig(5):Female patient, 55 years old. She underwent left BCS for left breast cancer 5 years ago. During routine follow up LIQ speculated dense mass lesion at the operative bed depicted by mammography.CESM: CESM in (A) MLO and (B) CC views of left breast showedtwo large irregular speculated masses, the largest seen related to skin retraction of surgical scar.The other in UIQ.B-MRI:BMRI in (C) and (D) post contrast subtraction images and (E)

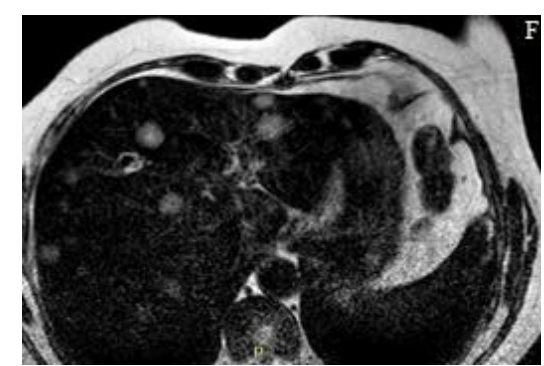

dynamic curve showed the left breast with multiple scattered variable sized irregular speculatedmass lesions andvariable pattern of enhancement in post contrast series. The largest lesion seen at the operative bed gave type III dynamic curve.Note that the number of the multiple lesions detected by MRI being much more than that detected by CESM.However some of these lesions proved by histopathology to be benignpathology revealingmulticenteric invasive ductal carcinoma. Yet few benign lesions were also noted.
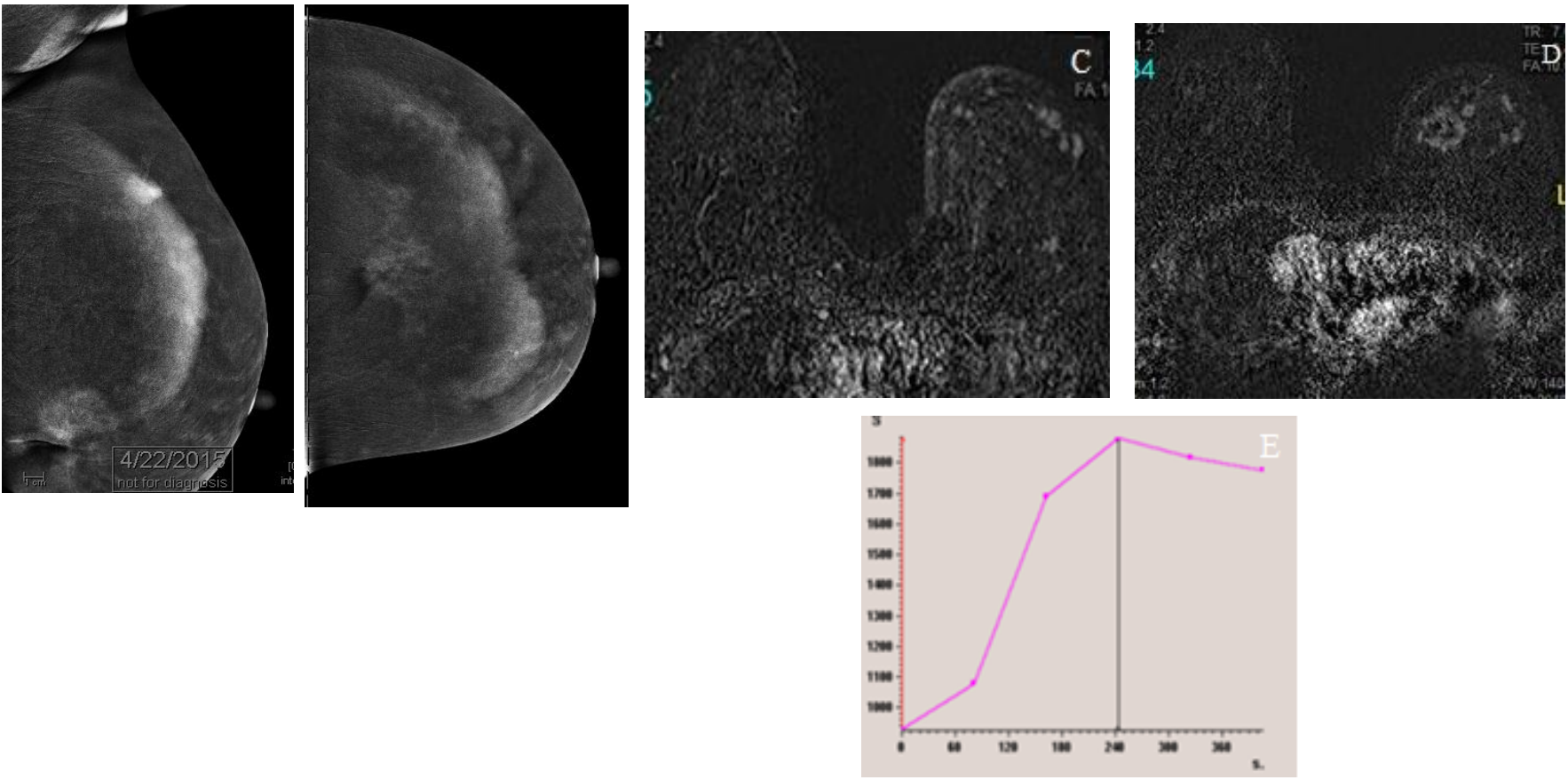

\section{Discussion}

Breast cancer is the most frequently diagnosed life-threatening cancer in women worldwide. It is accounting for $22.9 \%$ of all new female cancers and considered the most common cancer among women both in developed and developing countries. Breast cancer accounting for $37.7 \%$ of the total new cancer cases and it is the leading cause of cancer related mortality accounting for $29.1 \%$ of the cancer related deaths in Egypt $2008^{3}$. 
Previously, mastectomy was the standard operation, but recently breast conserving surgery (BCS; wide local excision or lumpectomy) has been becoming one of the primary surgical treatments.Several studies that compared the outcomes of BCS followed by whole-breast irradiation and mastectomy showed that the two operation techniques were equivalent regard to long-term survival if there were no contraindications ${ }^{5}$. Women with a history of breast cancer are at risk for developing local recurrences, a second ipsilateral breast cancer, or contralateral breast cancern ${ }^{6}$.

Up-to-date, mammography appears to be the most consistentmethod for the early detection of breast cancer; yet, it has bothlimited sensitivity and specificity in the detection and diagnosis of breast lesions, especially in dense breasts. Moreover thefull extent of the disease may not be clearly depicted. In reference to this, mammography misses about $20 \%$ of invasive breast cancers 2 .

The continued development of digital $\mathrm{x}$-ray systems enabledfurther techniques such as tomosynthesis and contrastenhancedmammography (CESM) to overcome thelimitations of MG. CESM involves a double exposure (lowandhighenergy x-rays), performed after intravenous injectionof an iodinated contrast agent. This resulted in a low-energyimage, comparable to mammography, as well as a recombinedimage, calculated from both low- and high-energy images,showing contrast agent uptake throughout the breast. Similarto breast MRI, contrast agent uptake is more pronounced inmalignant tumors, which facilitates cancer detection ${ }^{7}$.

However, three major clinical studies have been performed comparing CESM with BMRI Imaging in subjects with a newly diagnosed cancer. These studies typically evaluate the modalities for their ability to detect the index lesion, depict its extent, and find additional, separate lesions 8.
All these studies have confirmed that CESM has promising potential as a breast imaging tool, and superior to regular mammography and even match the diagnostic performance of breast MRI ${ }^{\mathbf{8}}$.

Our study studied 30 patient with 27 recurrent case, 24 identified by CESM and 26 identified by BMRI. Sensitivity for CESM and BMRI was $88.9 \%$ and $96.3 \%$ respectively. CESM was found to have a higher PPV and a lower false positive rate compared to BMRI. The false positive rate for CESM was $3.3 \%$ (1/30) versus $13.3 \%$ $(4 / 30,2 / 30$ from index lesions and $2 / 30$ in multicenteric cases) for BMRI. CESM had a PPV of $96.00 \%$ while PPV for BMRI was $92.86 \%$.The difference was not statistically significant $(P=0.62)$. MRI has aslightly higher level of accuracy than CESM 90\% vs. $86.6 \%$ respectively. The difference had no statistical significance $(\mathrm{P}=0.60)$.

The mean tumor sizes were as follows: CESM was about $2.66 \mathrm{~mm}$, MRI was $2.55 \mathrm{~mm}$ and post-surgical pathology was $2.49 \mathrm{~mm}$. The equivalence test showed that there was no statistically significant difference regarding size estimation between CEDM and pathology $(p=0.329)$, nor between MRI and pathology $(p=0.607)$. Finally there was also no statistically significant difference between size estimates in CEDM and MRI $(p=0.918)$ which matched with ${ }^{7,9}$.

The CESM is a potentially useful modality to distinguish between postoperative breast remodeling and cancer relapse $^{\mathbf{1 0 . 1 1}}$. Our study agreed with that, as we had 2 cases during routine follow up with mammography that showed area of architecture distortion.The absence of enhancement in CESM in one of them increased confidence of fibrotic tissue scar. BMRI also showed no enhancement and the histopathology confirmed the fibrotic scar. On the other hand, the $2^{\text {nd }}$ case, showed irregular intense marginal enhancement in CESM.While,BMRI showed seroma with thick irregular marginal enhancement raising 
the possibility of recurrence.By pathology, it was invasive ductal carcinoma.

Findings that were identified on low-energy images include microcalcifications, enhancing focus, and architectural distortion or speculated lesion, allowed CESM to detect additional lesions like low-grade or in situ tumors, which might be missed on both subtracted post contrast CESM and BMRI when there is no significant lesion enhancement ${ }^{\mathbf{1 2}}$. These agreed with ourstudy, as one of false negative results was a case with DCIS representedby cluster of malignant microcalcifications in low-energy image with no underlying mass enhancement in the combined image. MRI also failed to detect any mass enhancement. The associated micro-calcification in low- energy images gave alert to further assessment by stereotactic biopsy that confirmed the diagnosis of DCIS. Another case of a false negative result in CESM was patient with low cardiac output that had recurrence of invasive ductal carcinoma infiltrating the chest wall. It was missed by combined images but the low energy image showed the speculated mass lesion that was also detected in MRI images

MRI were superior in detection of additional lesions. It detected 9/9 (100\%) from multicenteric lesions comparing to $7 / 9$ (78\%) detected by CESM. However, the number of false positives of MRI was greater 2/9 (greater specificity of CESM) and the predictive value for carcinomas was significantly greater with CESM (100\% and $78 \%$ successively). This matched with ${ }^{\mathbf{8 , 9 , 1 0}}$

Patients with silicone surgical implants were usually tagged as not eligible for CESM diagnosis. verbal communication from General Electric Healthcare TM). They studied 8 patients where CESM only diagnosed cancer through in 50\% of all cases $^{\mathbf{1 0}}$. In our study, only one case was included. Although, recurrence was detected with similar result like MRI, yet MRI still had the upper hand in evaluating the implant integrity and detection of any intra or extra capsular rupture.

The breast MR imaging may be more advantageous compared to US as an adjunctive surveillance tool, in detection of the extra mammary malignancy. The Extra mammary cancerdefined as loco-regional disease (cancer in the ipsilateral axilla, internal mammary or supraclavicular lymph nodes or in the mastectomy bed) and distant metastasis $^{13}$. In our study,BMRI detected 9 cases of loco-regionalextra-mammary lesions, 5 case of recurrence in mastectomy bed one of them was infiltrating the chest wall , 3 cases of ipsilateral solid axillary lymph nodes as well as one case of supraclavicular solid lymph nodes. MRI also detected many cases of distant metastasis; two cases of pulmonary metastatic nodules, 3 cases of liver metastatic lesions, one case of dorsal vertebral metastasis and 2 cases of bilateral pleural effusion.

\section{Conclusion:}

This study demonstrates better lesion detection of CESM and BMRI than MG alone, with the largest benefit for patients with dense breasts. Despite the lower sensitivity, CESM showed greater specificity than BMRI; however both techniques displayed comparable accuracies for breast cancer detection and size estimation.

These results are promising as CESM appears to be a suitable alternative to BMRI to improve the detection of the breast cancer recurrence especially for patients with contraindications to BMRI as well as in regions with limited BMRI availability or lack of reimbursement

\section{References}

1. Sharma U, Gupta $S \quad R$ and Jagannathan N R (2008): Magnetic resonance imaging (MRI) and Spectroscopy (MRS) in breast cancer. Magnetic resonance insights, 2:93-108. 
2. Kamal RM ,Helal MH, Wessam R, Mansour SM, Godda I, Alieldin N (2015): Contrast-enhanced spectral mammography: Impact of the qualitative morphology descriptors on the diagnosis of breast lesions. European Journal of Radiology, 84 :049-1055.

3. Helal M , Abu Samra M, Ibraheem M, Salama A, Hassan E, and Hassan N (2017): Accuracy of CESM versus conventional mammography and ultrasound in evaluation of BI-RADS 3 and 4 breast lesions with pathological correlation. The Egyptian Journal of Radiology and Nuclear Medicine, 48: 741-750.

4. Mokhtar $O$ and Mahmoud $S$ (2014):Can contrast enhanced mammography solve the problem of dense breast lesions?.The Egyptian Journal of Radiology and Nuclear Medicine, 45:1043-1052.

5. Ditsatham C, SomwangprasertA, Watcharachan $K$, Wongmaneerung Ph, Khorana J (2016): Factors affecting local recurrence and distant metastases of invasive breast cancer after breast-conserving surgery in Chiang Mai University Hospital.Breast Cancer: Targets and Therapy, 8:47-52.

6. Drukteinis J S, Gombos EC and Raza $S$ (2012): MRI assessment of the breast after conservative therapy: distinguishing benign from malignant lesions. Radiographic, 32:219-234.

7. Fallenberge $M$, Schmitzbergerf $F$, Amer H, Heppner B, Balleyguier C, Diekmann F, EngelkenF, Mann RM, Renz RM, BickU, Hamm B, Dromain C (2017): Contrast-enhanced spectral mammography vs. mammography and MRI clinical performance in a multi- reader evaluation. EurRadiol, 27:27522764.

8. Lewin J (2017): Comparison of Contrast Enhanced Mammography and Contrast-Enhanced Breast MR Imaging. Magn Reson Imaging Clin N Am.,14:15.

9. Jochelson M, Dershaw D, Sung J (2013): Bilateral Contrast-enhanced Dual-Energy Digital Mammography: Feasibility and Comparison with Conventional Digital Mammography and MR Imaging in Women with Known Breast Carcinoma. Radiology, 266: 743-751.

10. Aja MT, Rodríguez $M \quad R \quad S$, Hernández A, Benítez VV, Luzardo O P (2014): Dual-energy contrastenhanced mammography. Radiology, 56(5):390-399.

11. Badr S, Laurent $\mathrm{N}$, Régis $\mathrm{C}$, Boulanger L, Lemaille S, Poncelet E (2014):Dual-energy contrast-enhanced digital mammography in routine clinical practice in 2013. Diagnostic and Interventional Imaging , 95: 245-258.

12. Li L., Roth R., Germaine P., Ren S., Lee M., Hunter K., Tinney E. and Liao L (2017): Contrast-enhanced spectral mammography (CESM) versus breast magnetic resonance imaging (MRI): A retrospective comparison in 66 breast lesions.Diagnostic and Interventional Imaging , 98: 113-123.

13. Park VY, Kim E, Kim M, Moon $\mathbf{H}$ and Yoon $J$ (2018):BMRI for surveillance of women with a personal history of breast cancer: outcomes stratifiedby interval between definitive surgery and surveillance MR imaging. BMC Cancer , 18(91) :1-8 